This is a preprint of an article accepted for publication in Graphs and Combinatorics (c) 2008 (copyright owner as specified in the journal). 


\title{
Further 6-sparse Steiner triple systems
}

\author{
A. D. Forbes ${ }^{1}$, M. J. Grannell ${ }^{2}$ and T. S. Griggs ${ }^{3}$ \\ Department of Mathematics, The Open University, Walton Hall, Milton Keynes MK7 6AA, UK. \\ 1 e-mail: anthony.d.forbes@gmail.com \\ 2 e-mail: m.j.grannell@open.ac.uk \\ 3 e-mail: t.s.griggs@open.ac.uk
}

\begin{abstract}
We give a construction that produces 6-sparse Steiner triple systems of order $v$ for all sufficiently large $v$ of the form $3 p, p$ prime and $p \equiv 3(\bmod 4)$. We also give a complete list of all 4296 -sparse systems with $v<10000$ produced by this construction.
\end{abstract}

Key words. Steiner triple system, 6-sparse, Pasch configuration, mitre configuration, crown configuration.

\section{Introduction}

A Steiner triple system of order $v, \operatorname{STS}(v)$, is a pair $(V, \mathcal{B})$ where $V$ is a set of cardinality $v$ of elements, or points, and $\mathcal{B}$ is a collection of triples, also called blocks, which has the property that every pair of distinct elements of $V$ occurs in precisely one triple. It is well known that an $\operatorname{STS}(v)$ exists if and only if $v \equiv 1$ or $3(\bmod 6)$. Such values are called admissible.

For any two points $a$ and $b$ in an $\operatorname{STS}(v),(V, \mathcal{B})$, we define the cycle graph $G_{a, b}$ as follows. The vertex set of $G_{a, b}$ is $V \backslash\{a, b, a * b\}$, where we denote by $x * y$ the third point in a block containing the pair $\{x, y\}$. The edge set of $G_{a, b}$ is the set of pairs $\{x, y\}$ such that either $\{x, y, a\}$ is a block or $\{x, y, b\}$ is a block. Clearly, $G_{a, b}$ is a set of disjoint cycles $\left\{C_{n_{1}}, C_{n_{2}}, \ldots, C_{n_{r}}\right\}$, where $n_{1}+n_{2}+\ldots+n_{r}=v-3$ and for $i=1,2, \ldots, r, n_{i}$ is even and $n_{i} \geq 4$.

A configuration in the context of a Steiner triple system is a set of triples, also called blocks, which has the property that every pair of distinct elements occurs in at most one triple. If $\mathcal{C}$ is a configuration, we denote by $P(\mathcal{C})$ its set of points. Two configurations $\mathcal{C}$ and $\mathcal{D}$ are said to be isomorphic, in symbols $\mathcal{C} \cong \mathcal{D}$, if there exists a bijection $\phi: P(\mathcal{C}) \rightarrow P(\mathcal{D})$ such that for each triple $T \in \mathcal{C}, \phi(T)$ is a triple in $\mathcal{D}$. For a Steiner triple system $(V, \mathcal{B})$, the set $\mathcal{B}$ itself may be regarded as a configuration with $P(\mathcal{B})=V$. The degree of a point in a configuration is the number of blocks of the configuration which contain that point. We sometimes write blocks with set brackets and commas omitted, so that for example $\{0,1,3\}$ might be written as 013 .

In this paper we will be concerned with configurations having $n$ blocks and $n+2$ points. Such configurations are of particular interest because of the following result proved in [4]. 
Table 1. Configurations having $n$ blocks and $n+2$ points, $4 \leq n \leq 6$.

\begin{tabular}{|llll|}
\hline$n$ & Name & Blocks & Comment \\
\hline 4 & Pasch & $012,034,135,245$ & \\
5 & mitre & $012,034,135,236,456$ & \\
5 & & $012,034,135,245,056$ & contains Pasch \\
6 & 6-cycle & $012,034,135,246,257,367$ & \\
6 & crown & $012,034,135,236,147,567$ & \\
6 & & $012,034,135,236,146,057$ & contains Pasch \\
6 & & $012,034,135,236,146,247$ & contains Pasch \\
6 & & $012,034,135,236,147,257$ & contains mitre \\
\hline
\end{tabular}

Theorem 1. For every integer $d \geq 3$ and for every integer $n$ satisfying $n \geq\left\lceil\frac{d}{2}\right\rceil$ there exists $v_{0}(n, d)$ such that for all admissible $v \geq v_{0}(n, d)$, every STS $(v)$ contains a configuration having $n$ blocks and $n+d$ points.

Here, the value of $d$ is sharp. For $d=2$, the theorem does not hold. Indeed, the case $d=2$ is the subject of a conjecture of Erdös [3]: For every integer $k \geq 4$, there exists $v_{0}(k)$ such that if $v>v_{0}(k)$ and if $v$ is admissible, then there exists an STS $(v)$ with the property that it contains no configurations having $n$ blocks and $n+2$ points for any $n$ satisfying $4 \leq n \leq k$. Such an $\operatorname{STS}(v)$ is said to be $k$-sparse. Clearly, a $k$-sparse system is also $k^{\prime}$-sparse for every $k^{\prime}$ satisfying $4 \leq k^{\prime} \leq k$.

Up to isomorphism, there is only one configuration having four blocks and six points, namely the Pasch configuration, also known as a quadrilateral; this is shown in Table 1. The existence of 4-sparse (better known as anti-Pasch) STS $(v)$ s for all admissible $v$, except $v=7$ and 13, was established in [1], [8], [10] and [7].

There is, up to isomorphism, only one Pasch-free configuration having five blocks and seven points, namely the mitre. This is also shown in Table 1. In [2], [9] and [5], culminating in recent work by Fujiwara and Wolfe [6], [12], it is established that anti-mitre systems exist for all admissible orders except $v=9$. Systems which are 5 -sparse, that is, both anti-Pasch and anti-mitre, are known for $v \equiv 1,19(\bmod 54)$, except possibly $v=109$, and for many other sporadic values [9], [6]. Also we are aware that there exists a 5sparse STS(109) [13]. Substantial further progress has recently been made by Wolfe in [14], where it is shown that 5-sparse $\operatorname{STS}(v)$ s exist for almost all admissible $v$ (meaning arithmetic set density 1 in the set of all admissible orders), and in [15], where existence for all $v \equiv 3(\bmod 6)$ with $v \geq 21$ is established.

There are, up to isomorphism, two Pasch-free and mitre-free configurations having six blocks and eight points, of which one is the 6-cycle. The other configuration is called the crown, a word that is suggested by following diagram.

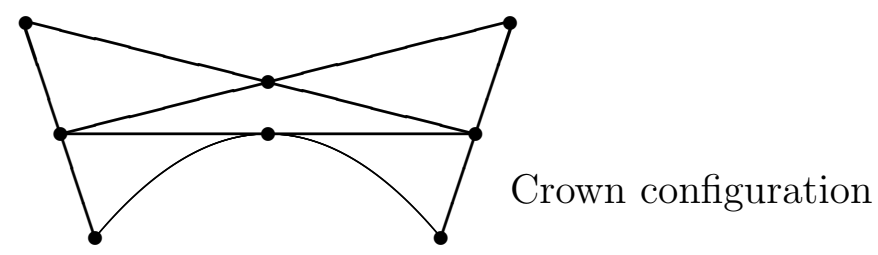

Thus a system is 6-sparse if and only if it contains no Pasch configurations, no mitres, no 6-cycles and no crowns. In [4] we presented the first known non-trivial examples of 6-sparse Steiner triple systems. Our results depended on two basic theorems. The first of these is the following. 
Theorem 2. Suppose that $v$ is a prime congruent to 7 modulo 12 and that $\chi$ is a multiplicative character of $G F(v)$ of order 6 . Suppose also that $\alpha \in G F(v)$ has the property that $\chi(\alpha) \neq-1,0,1$ and that $\chi(1-\alpha) \chi(\alpha)= \pm 1$. Let $G$ denote the group comprising all mappings on $G F(v)$ having the form $x \rightarrow a x+b$ for $a, b \in G F(v)$ with $\chi(a)=1$. Then the orbit generated by the block $\{0,1, \alpha\}$ under the action of $G$ forms a block transitive $S T S(v)$.

Using Theorem 2, we obtained 29 6-sparse systems with 27 different prime orders $v \equiv$ 7 (mod 12). Furthermore, by employing Weil's theorem on bounding character sums [11, page 43] we were able to show that our list of such systems is complete.

The other theorem from [4] asserts that the standard product construction preserves 6-sparseness under certain conditions.

Theorem 3. Suppose that $S=(V, \mathcal{B})$ is a block transitive Steiner triple system of order $v$, with $\alpha$ and $\chi$ as in Theorem 2 and $V=G F(v)$. Suppose also that $S^{*}=\left(W, \mathcal{B}^{*}\right)$ is a Steiner triple system of order $w$. For each block of $\mathcal{B}^{*}$, arbitrarily fix the order of the points, so that $\mathcal{B}^{*}$ may be regarded as a set of ordered triples $(i, j, k)$. Put $V^{\prime}=V \times W$ and let

$$
\begin{aligned}
\mathcal{B}^{\prime}= & \left\{\left\{a_{i}, b_{i}, c_{i}\right\}:\{a, b, c\} \in \mathcal{B}, i \in W\right\} \\
& \cup\left\{\left\{x_{i}, y_{j},(x \beta+y \gamma)_{k}\right\}: x, y \in G F(v),(i, j, k) \in \mathcal{B}^{*}\right\},
\end{aligned}
$$

where $\beta, \gamma \neq 0$ are fixed parameters in $G F(v)$. Then $S^{\prime}=\left(V^{\prime}, \mathcal{B}^{\prime}\right)$ is a Steiner triple system of order vw. Furthermore, if both $S$ and $S^{*}$ are 6 -sparse, if

$$
\alpha^{2} \notin\{\alpha-1,1-\alpha, \alpha+1,3 \alpha-1\}
$$

and if $\chi(\beta), \chi(\gamma), \chi(\beta / \gamma) \neq \pm 1$, then $S^{\prime}$ is also 6-sparse.

Having shown that (1) holds for each of our original 29 block transitive systems, we can repeatedly apply Theorem 3 , choosing, for example, $\beta=\alpha$ and $\gamma=1 / \alpha$, to establish that there are infinitely many 6-sparse Steiner triple systems.

In this paper we prove a theorem analogous to Theorem 2 for the case $v=3 p$, where $p$ is prime and $p \equiv 3(\bmod 4)$. Using this theorem we are able to construct 6 -sparse Steiner triple systems of order $3 p$ for all sufficiently large primes $p \equiv 3(\bmod 4)$.

\section{Steiner triple systems with $v \equiv 9(\bmod 12)$}

For the remainder of the paper, $p$ will always denote a prime congruent to 3 modulo 4 , and $\theta$ will denote the quadratic character modulo $p$. Thus if $x \not \equiv 0(\bmod p), \theta(x)=(x / p)$, the Legendre symbol, and if $x \equiv 0(\bmod p), \theta(x)=0$.

Theorem 4. Let $p=2 s+1 \geq 7$ be a prime such that $p \equiv 3(\bmod 4)$ and let $v=3 p$. Let $\tau$ be an integer modulo $v$ such that $\tau \not \equiv 0(\bmod 3)$ and $\tau$ is a primitive root modulo $p$. Let $\omega=\tau^{2} \bmod v$. Choose $\alpha$ modulo $v$ such that either $(i) \alpha \equiv 0(\bmod 3)$ and $\theta(\alpha-1)=1$, or $($ ii) $\alpha \equiv 1(\bmod 3)$ and $\theta(-\alpha)=1$. Then, with all arithmetic modulo $v$,

$$
\begin{array}{r}
\left\{\left\{m, m+\omega^{i}, m+\alpha \omega^{i}\right\}: i=0,1, \ldots, s-1, m=0,1, \ldots, v-1\right\} \\
\cup\left\{\left\{n, n+\frac{1}{3} v, n+\frac{2}{3} v\right\}: n=0,1, \ldots, \frac{1}{3} v-1\right\}
\end{array}
$$


is the set of blocks of an $S T S(v)$, defined on $\{0,1, \ldots, v-1\}$, which is generated by $\{0,1, \alpha\}$ and $\{0, v / 3,2 v / 3\}$ under the action of the group of mappings

$$
G=\left\{x \mapsto \omega^{i} x+m \bmod v, i=0,1, \ldots, s-1, m=0,1, \ldots, v-1\right\} .
$$

Proof. In this proof and the remarks which follow we shall tacitly assume that unless otherwise specified all arithmetic is performed modulo $v$.

Clearly, the orbit of the starter block $\{0, p, 2 p\}$ under the action of $G$ is $\{\{n, n+p, n+$ $2 p\}: n=0,1, \ldots, p-1\}$. Let

$$
\Omega(x)=\left\{x \omega^{i} \bmod v: i=0,1, \ldots, s-1\right\}
$$

and observe that for any $x$ modulo $v$, we have

$$
\theta(x \omega)=\theta(x), \quad x \omega \equiv x(\bmod 3)
$$

and

$$
\Omega(x)=\{y \bmod v: \theta(y)=\theta(x) \text { and } y \equiv x(\bmod 3)\} .
$$

Therefore we can prove the theorem by showing that the six differences $\pm 1, \pm \alpha$ and $\pm(1-\alpha)$ generated by the triple $\{0,1, \alpha\}$ have distinct combinations of quadratic character modulo $p$ and residue class modulo 3 . Since $\theta(-1)=-1$, this is possible if and only if $\alpha$ satisfies (i) or (ii) in the statement of the theorem.

The choice of $\tau$ is immaterial, subject to $\tau \not \equiv 0(\bmod 3)$ and $\tau$ being a primitive root modulo $p$. To see this, suppose $\tau^{\prime} \not \equiv 0(\bmod 3)$ is also a primitive root modulo $p$ and let $\omega^{\prime}=\left(\tau^{\prime}\right)^{2}$. Then $\tau^{\prime} \equiv \tau^{t}(\bmod p)$ for some $t$ with $(t, p-1)=1$ and it is plain that for any $x$,

$$
\Omega(x)=\left\{x\left(\omega^{\prime}\right)^{i} \bmod v: i=0,1, \ldots, s-1\right\} .
$$

If $\alpha \equiv 0(\bmod 3)$, the four $\operatorname{STS}(v)$ s generated by the blocks $\{0,1, \delta\}$ and $\{0, v / 3,2 v / 3\}$ for $\delta \in\{\alpha, 1-\alpha, 1 /(1-\alpha), 1-1 /(1-\alpha)\}$ are isomorphic under the mappings $x \mapsto 1-x$, $x \mapsto(x-1) /(\alpha-1)$ and $x \mapsto(\alpha-x) /(\alpha-1)$. If $\alpha \equiv 1(\bmod 3)$, the four STS $(v) \mathrm{s}$ generated by the blocks $\{0,1, \delta\}$ and $\{0, v / 3,2 v / 3\}$ for $\delta \in\{\alpha, 1-\alpha, 1 / \alpha, 1-1 / \alpha\}$ are isomorphic under the mappings $x \mapsto 1-x, x \mapsto x / \alpha$ and $x \mapsto 1-x / \alpha$.

The above observations may be used to reduce the size of a search for 6-sparse systems obtained from Theorem 4. A complete list, up to isomorphism, of such 6-sparse Steiner triple systems for $v<10000$ is given in Table 2. Systems with the same value of $v$ are pairwise non-isomorphic, as can be seen by examining the structure of the cycle graphs $G_{0,1}, G_{0, \alpha}, G_{1, \alpha}$ and $G_{0, v / 3}$. We refer to a system created by Theorem 4 as a two-generator system with parameters $v$ and $\alpha$.

The special mitres and Pasch configurations that are shown in [4] to be unavoidable in all systems with sufficiently large order obtained from Theorem 2 do not form in the twogenerator systems of Theorem 4 . We now prove that there is no such blocking mechanism to prevent the formation of 6-sparse two-generator systems of arbitrarily large orders.

Theorem 5. For all sufficiently large $v$ with $v=3 p, p$ prime and $p \equiv 3(\bmod 4)$, there exists $\alpha$ such that the two-generator system of Theorem 4 with parameters $v$ and $\alpha$ is 6-sparse. 
A. D. Forbes, M. J. Grannell and T. S. Griggs

Table 2. 6 -sparse systems with $v \equiv 9(\bmod 12)$

\begin{tabular}{|c|c|c|c|c|c|c|c|c|c|c|c|c|c|}
\hline$v$ & $\alpha$ & $v$ & $\alpha$ & $v$ & $\alpha$ & $v$ & $\alpha$ & $v$ & $\alpha$ & $v$ & $\alpha$ & $v$ & $\alpha$ \\
\hline 489 & 135 & 3837 & 880 & 5277 & 1377 & 6429 & 129 & 7977 & 1960 & 8637 & 919 & 9357 & 18 \\
\hline 501 & 160 & 3849 & 1263 & 5277 & 1486 & 6429 & 1462 & 7977 & 2404 & 8637 & 1393 & 9357 & 390 \\
\hline 1077 & 75 & 3909 & 544 & 5277 & 2074 & 6429 & 2097 & 7977 & 2944 & 8637 & 2046 & 9357 & 403 \\
\hline 1101 & 379 & 3909 & 1063 & 5349 & 15 & 6537 & 915 & 7989 & 402 & 8637 & 2077 & 9357 & 1033 \\
\hline 1149 & 328 & 3981 & 1627 & 5361 & 835 & 6537 & 1068 & 7989 & 657 & 8637 & 4141 & 9357 & 1516 \\
\hline 1329 & 309 & 4101 & 265 & 5361 & 1075 & 6609 & 31 & 7989 & 2298 & 8661 & 490 & 9357 & 2152 \\
\hline 1437 & 12 & 4101 & 427 & 5361 & 1377 & 6609 & 810 & 7989 & 3429 & 8661 & 1011 & 9357 & 2403 \\
\hline 1461 & 13 & 4101 & 561 & 5469 & 84 & 6717 & 954 & 8013 & 348 & 8661 & 1254 & 9357 & 2643 \\
\hline 1461 & 42 & 4281 & 204 & 5469 & 415 & 6753 & 1551 & 8013 & 496 & 8661 & 1918 & 9489 & 1048 \\
\hline 1509 & 490 & 4317 & 201 & 5469 & 1114 & 6753 & 2184 & 8013 & 549 & 8661 & 2901 & 9489 & 1191 \\
\hline 1569 & 232 & 4317 & 432 & 5469 & 1516 & 6861 & 385 & 8013 & 793 & 8709 & 42 & 9489 & 1809 \\
\hline 1641 & 223 & 4317 & 658 & 5493 & 430 & 6861 & 604 & 8013 & 1009 & 8709 & 99 & 9489 & 4314 \\
\hline 1689 & 276 & 4317 & 693 & 5493 & 1576 & 6933 & 933 & 8013 & 2353 & 8709 & 250 & 9501 & 168 \\
\hline 1857 & 141 & 4317 & 744 & 5541 & 1104 & 6933 & 3030 & 8049 & 570 & 8709 & 705 & 9501 & 471 \\
\hline 1857 & 328 & 4317 & 993 & 5541 & 1707 & 7017 & 81 & 8049 & 1173 & 8709 & 1296 & 9501 & 486 \\
\hline 1929 & 502 & 4353 & 660 & 5541 & 2344 & 7017 & 240 & 8061 & 3 & 8709 & 1395 & 9501 & 1605 \\
\hline 1929 & 508 & 4353 & 1057 & 5601 & 1065 & 7017 & 1117 & 8061 & 18 & 8709 & 1695 & 9501 & 2514 \\
\hline 1941 & 3 & 4377 & 58 & 5613 & 1470 & 7041 & 351 & 8061 & 57 & 8709 & 2010 & 9501 & 3609 \\
\hline 1941 & 736 & 4377 & 184 & 5613 & 1900 & 7041 & 1009 & 8061 & 439 & 8709 & 3925 & 9561 & 148 \\
\hline 1977 & 519 & 4377 & 409 & 5613 & 2218 & 7041 & 1305 & 8061 & 576 & 8781 & 366 & 9561 & 4164 \\
\hline 2157 & 36 & 4449 & 94 & 5613 & 2343 & 7041 & 1392 & 8061 & 1270 & 8781 & 498 & 9561 & 4273 \\
\hline 2157 & 186 & 4497 & 430 & 5637 & 880 & 7053 & 520 & 8061 & 1333 & 8781 & 685 & 9573 & 54 \\
\hline 2181 & 9 & 4569 & 370 & 5721 & 1594 & 7053 & 985 & 8061 & 1531 & 8781 & 979 & 9573 & 162 \\
\hline 2217 & 193 & 4569 & 1402 & 5853 & 376 & 7053 & 1650 & 8097 & 666 & 8781 & 2251 & 9573 & 391 \\
\hline 2229 & 880 & 4569 & 1837 & 5853 & 435 & 7053 & 2227 & 8121 & 307 & 8781 & 3706 & 9573 & 687 \\
\hline 2361 & 979 & 4593 & 117 & 5853 & 1677 & 7113 & 2404 & 8121 & 1231 & 8817 & 571 & 9573 & 1093 \\
\hline 2433 & 594 & 4593 & 1210 & 5853 & 2064 & 7149 & 714 & 8121 & 1347 & 8817 & 1552 & 9573 & 1350 \\
\hline 2589 & 684 & 4629 & 366 & 5937 & 1365 & 7197 & 966 & 8133 & 292 & 8817 & 1969 & 9573 & 2085 \\
\hline 2649 & 421 & 4629 & 1699 & 5937 & 1606 & 7197 & 1138 & 8133 & 1386 & 8817 & 2991 & 9573 & 2202 \\
\hline 2649 & 609 & 4677 & 12 & 5961 & 358 & 7233 & 1794 & 8133 & 2764 & 8913 & 694 & 9609 & 306 \\
\hline 2721 & 534 & 4677 & 78 & 5961 & 1540 & 7269 & 85 & 8133 & 3225 & 8913 & 1725 & 9609 & 721 \\
\hline 2733 & 24 & 4677 & 99 & 5997 & 643 & 7341 & 1390 & 8157 & 2062 & 8913 & 3289 & 9609 & 1191 \\
\hline 2733 & 240 & 4677 & 126 & 5997 & 1372 & 7341 & 1597 & 8193 & 160 & 8997 & 150 & 9609 & 1260 \\
\hline 2733 & 585 & 4677 & 583 & 6009 & 360 & 7377 & 891 & 8193 & 1153 & 8997 & 351 & 9609 & 1731 \\
\hline 2733 & 682 & 4677 & 1240 & 6009 & 900 & 7377 & 2287 & 8301 & 700 & 8997 & 367 & 9609 & 1783 \\
\hline 2841 & 447 & 4701 & 76 & 6009 & 1167 & 7401 & 87 & 8301 & 835 & 8997 & 753 & 9609 & 2994 \\
\hline 2949 & 711 & 4701 & 337 & 6033 & 126 & 7401 & 3546 & 8301 & 871 & 8997 & 955 & 9609 & 3166 \\
\hline 2949 & 906 & 4701 & 43 & 6033 & 792 & 7509 & 907 & 8301 & 994 & 8997 & 1227 & 9753 & 2193 \\
\hline 2949 & 919 & 4701 & 499 & 6033 & 2251 & 7509 & 1293 & 8301 & 1011 & 8997 & 2253 & 9753 & 3313 \\
\hline 2973 & 288 & 4749 & 418 & 6081 & 457 & 7509 & 1762 & 8301 & 2398 & 8997 & 2857 & 9753 & 3454 \\
\hline 2973 & 309 & 4749 & 1239 & 6081 & 1360 & 7593 & 103 & 8373 & 537 & 8997 & 3295 & 9777 & 364 \\
\hline 3057 & 954 & 4749 & 1294 & 6117 & 604 & 7593 & 219 & 8373 & 1657 & 8997 & 3606 & 9777 & 903 \\
\hline 3093 & 445 & 4821 & 43 & 6117 & 2373 & 7593 & 1108 & 8373 & 2697 & 9033 & 273 & 9813 & 1743 \\
\hline 3093 & 610 & 4821 & 565 & 6117 & 2490 & 7617 & 85 & 8373 & 2913 & 90 & 1582 & 9813 & 3049 \\
\hline 3117 & 345 & 4821 & 826 & 6189 & 63 & 7617 & 223 & 8409 & 630 & 9033 & 3421 & 9897 & 1206 \\
\hline 3117 & 579 & 4821 & 1240 & 6189 & 1429 & 7617 & 231 & 8409 & 1927 & 9057 & 397 & 9921 & 96 \\
\hline 3189 & 318 & 4821 & 1587 & 6189 & 2224 & 7617 & 816 & 8409 & 2554 & 9057 & 720 & 9921 & 910 \\
\hline 3261 & 9 & 4857 & 163 & 6249 & 69 & 7617 & 864 & 8457 & 685 & 9057 & 1308 & 9921 & 3514 \\
\hline 3261 & 409 & 4857 & 1057 & 6249 & 561 & 7629 & 141 & 8529 & 57 & 9057 & 2643 & 9921 & 3865 \\
\hline 3261 & 735 & 4881 & 942 & 6249 & 2653 & 7629 & 876 & 8529 & 471 & 9069 & 2761 & 9957 & 99 \\
\hline 3309 & 390 & 4881 & 1761 & 6261 & 907 & 7653 & 162 & 8529 & 507 & 9201 & 486 & 9957 & 144 \\
\hline 3309 & 940 & 4989 & 336 & 6261 & 1200 & 7653 & 366 & 8529 & 3192 & 9201 & 595 & 9957 & 2194 \\
\hline 3453 & 802 & 5001 & 919 & 6261 & 1219 & 7653 & 498 & 8553 & 444 & 9201 & 946 & 9957 & 4138 \\
\hline 3513 & 223 & 5001 & 1530 & 6261 & 1422 & 7653 & 1440 & 8553 & 568 & 9201 & 1327 & 9969 & 619 \\
\hline 3513 & 598 & 5001 & 1608 & 6297 & 286 & 7653 & 1612 & 8553 & 1189 & 9201 & 2146 & 9969 & 2410 \\
\hline 3561 & 313 & 5097 & 70 & 6297 & 1278 & 7737 & 1192 & 8553 & 1738 & 9201 & 2365 & 9969 & 2565 \\
\hline 3669 & 87 & 5097 & 633 & 6297 & 1983 & 7773 & 1327 & 8553 & 2931 & 9237 & 648 & 9993 & 2443 \\
\hline 3669 & 231 & 5097 & 1227 & 6333 & 135 & 7773 & 2185 & 8637 & 52 & 9237 & 693 & & \\
\hline 3669 & 520 & 5097 & 1747 & & 648 & 7773 & 2239 & 8637 & 232 & 9237 & 2287 & & \\
\hline 3693 & 102 & 5169 & 915 & 6333 & 810 & 7773 & 3270 & 8637 & 432 & 9249 & 556 & & \\
\hline 3693 & 544 & 5241 & 538 & 6333 & 2242 & 7941 & 1864 & 8637 & 523 & 9249 & 3069 & & \\
\hline 3693 & 838 & 5241 & 2160 & 6429 & 72 & 7977 & 1107 & 8637 & 744 & 9249 & 3339 & & \\
\hline
\end{tabular}


The proof of this theorem makes use of the following lemmas, the last of which relies on extensive computations.

Lemma 1. Let $n$ be a positive integer, let $p$ be a prime, let

$$
\begin{gathered}
a_{1,1} x_{1}+a_{1,2} x_{2}+\ldots+a_{1, n} x_{n} \equiv c_{1}(\bmod p) \\
a_{2,1} x_{1}+a_{2,2} x_{2}+\ldots+a_{2, n} x_{n} \equiv c_{2}(\bmod p) \\
\ldots \\
a_{n, 1} x_{1}+a_{n, 2} x_{2}+\ldots+a_{n, n} x_{n} \equiv c_{n}(\bmod p)
\end{gathered}
$$

be a set of linear congruences modulo $p$ and let $\mathbf{A}=\left[a_{i, j}\right]$ be the corresponding matrix of coefficients. Suppose $|\mathbf{A}| \not \equiv 0(\bmod p)$. Then there exists a unique solution of (2) in $G F(p)$. Furthermore, the solution is formally the same as that obtained by solving (2) over the rationals.

Proof. This is well known.

Lemma 2. Let $S=(V, \mathcal{B})$ be a two-generator Steiner triple system with parameters $v$ and $\alpha$ containing one of the configurations Pasch, mitre, 6 -cycle, crown. Let $V=\{0,1, \ldots, v-$ $1\}$ and let $\Gamma=\left\{\mathcal{G}_{1}, \mathcal{G}_{2}, \ldots, \mathcal{G}_{13}\right\}$, where

$$
\begin{aligned}
& \mathcal{G}_{1}=\left\{\{0,1, \alpha\},\left\{0, x_{1}, x_{2}\right\},\left\{1, x_{2}, x_{3}\right\},\left\{\alpha, x_{1}, x_{3}\right\}\right\}, \\
& \mathcal{G}_{2}=\left\{\{0,1, \alpha\},\left\{0, x_{1}, x_{2}\right\},\left\{0, x_{3}, x_{4}\right\},\left\{1, x_{1}, x_{3}\right\},\left\{\alpha, x_{2}, x_{4}\right\}\right\}, \\
& \mathcal{G}_{3}=\left\{\{0,1, \alpha\},\left\{1, x_{1}, x_{2}\right\},\left\{1, x_{3}, x_{4}\right\},\left\{0, x_{1}, x_{3}\right\},\left\{\alpha, x_{2}, x_{4}\right\}\right\}, \\
& \mathcal{G}_{4}=\left\{\{0,1, \alpha\},\left\{\alpha, x_{1}, x_{2}\right\},\left\{\alpha, x_{3}, x_{4}\right\},\left\{0, x_{1}, x_{3}\right\},\left\{1, x_{2}, x_{4}\right\}\right\}, \\
& \mathcal{G}_{5}=\left\{\{0,1, \alpha\},\left\{0, x_{1}, x_{2}\right\},\left\{0, x_{3}, x_{4}\right\},\left\{x_{5}, 1, x_{1}\right\},\left\{x_{5}, \alpha, x_{3}\right\},\left\{x_{5}, x_{2}, x_{4}\right\}\right\}, \\
& \mathcal{G}_{6}=\left\{\{0,1, \alpha\},\left\{1, x_{1}, x_{2}\right\},\left\{1, x_{3}, x_{4}\right\},\left\{x_{5}, 0, x_{1}\right\},\left\{x_{5}, \alpha, x_{3}\right\},\left\{x_{5}, x_{2}, x_{4}\right\}\right\}, \\
& \mathcal{G}_{7}=\left\{\{0,1, \alpha\},\left\{\alpha, x_{1}, x_{2}\right\},\left\{\alpha, x_{3}, x_{4}\right\},\left\{x_{5}, 0, x_{1}\right\},\left\{x_{5}, 1, x_{3}\right\},\left\{x_{5}, x_{2}, x_{4}\right\}\right\}, \\
& \mathcal{G}_{8}=\left\{\{0,1, \alpha\},\left\{0, x_{1}, x_{2}\right\},\left\{0, x_{3}, x_{5}\right\},\left\{1, x_{1}, x_{4}\right\},\left\{\alpha, x_{1}, x_{5}\right\},\left\{x_{2}, x_{3}, x_{4}\right\}\right\}, \\
& \mathcal{G}_{9}=\left\{\{0,1, \alpha\},\left\{0, x_{1}, x_{2}\right\},\left\{0, x_{3}, x_{5}\right\},\left\{\alpha, x_{1}, x_{4}\right\},\left\{1, x_{1}, x_{5}\right\},\left\{x_{2}, x_{3}, x_{4}\right\}\right\}, \\
& \mathcal{G}_{10}=\left\{\{0,1, \alpha\},\left\{1, x_{1}, x_{2}\right\},\left\{1, x_{3}, x_{5}\right\},\left\{0, x_{1}, x_{4}\right\},\left\{\alpha, x_{1}, x_{5}\right\},\left\{x_{2}, x_{3}, x_{4}\right\}\right\}, \\
& \mathcal{G}_{11}=\left\{\{0,1, \alpha\},\left\{1, x_{1}, x_{2}\right\},\left\{1, x_{3}, x_{5}\right\},\left\{\alpha, x_{1}, x_{4}\right\},\left\{0, x_{1}, x_{5}\right\},\left\{x_{2}, x_{3}, x_{4}\right\}\right\}, \\
& \mathcal{G}_{12}=\left\{\{0,1, \alpha\},\left\{\alpha, x_{1}, x_{2}\right\},\left\{\alpha, x_{3}, x_{5}\right\},\left\{0, x_{1}, x_{4}\right\},\left\{1, x_{1}, x_{5}\right\},\left\{x_{2}, x_{3}, x_{4}\right\}\right\}, \\
& \mathcal{G}_{13}=\left\{\{0,1, \alpha\},\left\{\alpha, x_{1}, x_{2}\right\},\left\{\alpha, x_{3}, x_{5}\right\},\left\{1, x_{1}, x_{4}\right\},\left\{0, x_{1}, x_{5}\right\},\left\{x_{2}, x_{3}, x_{4}\right\}\right\} .
\end{aligned}
$$

Then there is a $\mathcal{G} \in \Gamma$ such that $\mathcal{G} \subset \mathcal{B}$ for some $x_{1}, x_{2}, \ldots, x_{n} \in V$, where $n=|\mathcal{G}|-1$.

Proof. By Theorem $4, v=3 p, p$ prime, $p \equiv 3(\bmod 4)$, and $S$ is generated by blocks $\{0,1, \alpha\}$ and $\{0, p, 2 p\}$. Let $\mathcal{X}$ be one of the configurations Pasch, mitre, 6-cycle, crown. Suppose $\mathcal{X} \subset \mathcal{B}$.

Observe that $\mathcal{G}_{1}$ is a Pasch configuration, $\mathcal{G}_{2}, \mathcal{G}_{3}$ and $\mathcal{G}_{4}$ are mitres, $\mathcal{G}_{5}, \mathcal{G}_{6}$ and $\mathcal{G}_{7}$ are 6-cycles, $\mathcal{G}_{8}, \mathcal{G}_{9}, \ldots, \mathcal{G}_{13}$ are crowns and the block of $\mathcal{G} \in \Gamma$ labelled $\{0,1, \alpha\}$ is one of two intersecting blocks which map to each other under an automorphism of $\mathcal{G}$. Since $\mathcal{X}$ cannot contain two intersecting blocks belonging to the orbit of $\{0, p, 2 p\}$, it is straightforward to verify (perhaps by drawing diagrams) that there exists an automorphism of $S$ which maps $\mathcal{X}$ to some $\mathcal{G} \in \Gamma$ for some $x_{1}, x_{2}, \ldots, x_{|\mathcal{G}|-1} \in V$.

Lemma 3. Let $p$ be prime and suppose that the polynomial $f(x)$ is not a constant multiple of a square over $G F(p)$. Then

$$
\left|\sum_{x \in G F(p)} \theta(f(x))\right|=O(\sqrt{p}) .
$$


Proof. This is a special case of the theorem on page 43 of [11].

In the next lemma we introduce a set of polynomials, $\Lambda$. In Lemma 5 we investigate certain sets of linear congruences. The coefficients of these congruences involve a parameter, $\alpha$. We wish to show that there exists an $\alpha$ such that either the congruences have no solution, or the solution satisfies certain conditions that can be expressed in the form $\theta(\rho(\alpha))=1$ for certain rational functions $\rho(x)$. We then find that there is a set of polynomials $\Lambda$ with the property that if $\theta(\lambda(\alpha))=1$ for all $\lambda(x) \in \Lambda$, then $\theta(\rho(\alpha))=-1$ for at least one of the functions $\rho(x)$. Actually, to deal with questions of existence and uniqueness of solutions, slightly more than this is required, and the key property of $\Lambda$ is that given in Lemma 4 . The set $\Lambda$ given in this lemma was obtained by considering the numerators and denominators of the functions $\rho(x)$. It is not feasible to explain why each individual polynomial is included in $\Lambda$. However, we give below, following the proof of Lemma 5, several examples to illustrate the method. In particular, Example 1 explains why $-x^{3}+5 x^{2}-6 x+3 \in \Lambda$.

Lemma 4. Let

$$
\begin{aligned}
\Lambda= & \left\{x, x-1, x+1,-2 x+1,2 x-3,-x+3, x^{2}+1,-x^{2}-2,\right. \\
& -x^{2}-x+1, x^{2}-x+1,-x^{2}+x+1,-x^{2}+2 x-2, \\
& -x^{2}+3 x-3,-2 x^{2}+3 x-2,3 x^{2}-4 x+2,2 x^{2}-4 x+3, \\
& -2 x^{2}+3 x-3,3 x^{2}-5 x+3, x^{2}-2 x+3, x^{2}-3 x+1, \\
& -x^{3}+x^{2}-1,-x^{3}+2 x^{2}-x-1,-x^{3}+3 x^{2}-2 x+1, \\
& x^{3}-2 x^{2}+3 x-3, x^{3}-3 x^{2}+6 x-3, x^{3}-3 x+3, \\
& \left.-x^{3}+5 x^{2}-6 x+3,-x^{3}+3 x^{2}-4 x+1\right\} .
\end{aligned}
$$

Given any positive number $N$, for all sufficiently large prime $p$, there exist at least $N$ numbers $\alpha$, distinct modulo $p$, such that $\theta(\lambda(\alpha))=1$ for all $\lambda(x) \in \Lambda$.

Proof. Let

$$
\pi(x)=\prod_{\lambda(x) \in \Lambda}(1+\theta(\lambda(x)))
$$

and

$$
\Delta=\sum_{x \in \mathrm{GF}(p)} \pi(x)
$$

Then

$$
\Delta=p+\sum_{f(x)} \sum_{x \in \mathrm{GF}(p)} \theta(f(x))
$$

where $f(x)$ in the outer sum runs through all $2^{|\Lambda|}-1$ non-empty products of polynomials $\lambda(x) \in \Lambda$. It is easily checked that over the rationals the polynomial $\prod_{\lambda(x) \in \Lambda} \lambda(x)$ has nonzero discriminant. Hence, assuming that $p$ is sufficiently large, $f(x)$ is never a constant multiple of a square over $\operatorname{GF}(p)$. So by Lemma 3 we have $\Delta=p-O(\sqrt{p})$.

Since both $\pi(x)$ and the number of factors of $\pi(x)$ which are equal to 1 are bounded as $p \rightarrow \infty$, it follows that for each fixed $N$ and for $p$ sufficiently large, there exist $N$ distinct values of $\alpha$ modulo $p$ such that $\theta(\lambda(\alpha))=1$ for all $\lambda(x) \in \Lambda$. 
Lemma 5. Let $v=3 p, p$ prime, $p \equiv 3(\bmod 4)$. Let $\Lambda$ be the set of polynomials in Lemma 4. Then there exists a polynomial $Q(x)$ such that if $\alpha \equiv 0(\bmod 3)$, if $\theta(\lambda(\alpha))=1$ for all $\lambda(x) \in \Lambda$ and if $Q(\alpha) \not \equiv 0(\bmod p)$, then there exists a 6-sparse two-generator Steiner triple system with parameters $v$ and $\alpha$.

Proof. Let $v=3 p, p$ prime, $p \equiv 3(\bmod 4)$ and suppose $\alpha$ satisfies the conditions of the lemma with $Q(x)$ to be chosen later. Observe that $x-1 \in \Lambda$; therefore $\theta(\alpha-1)=1$, as required by Theorem 4, and hence there exists a two-generator Steiner triple system $S=(V, \mathcal{B})$ with parameters $v$ and $\alpha$. We show that with a suitable choice of $Q(x) S$ is 6-sparse.

Let $\Gamma=\left\{\mathcal{G}_{1}, \mathcal{G}_{2}, \ldots, \mathcal{G}_{13}\right\}$ be the set of configurations in Lemma 2. Let $\mathcal{G} \in \Gamma$ and let $\mathcal{G}$ have $n+1$ blocks. For $d=1,2, \ldots, n$, let $\left(a_{d}, b_{d}, c_{d}\right)$ be the $d$ th block of $\mathcal{G} \backslash\{\{0,1, \alpha\}\}$ in some ordering. Then if $\mathcal{G} \subset \mathcal{B}$, we have the following set of $3 n$ linear congruences modulo $3 p$ in variables $x_{1}, x_{2}, \ldots, x_{n}, m_{1}, m_{2}, \ldots, m_{n}$ and the variables $\omega_{d}$ for those $d$ where the corresponding congruences have the first alternative on the right:

$$
\begin{aligned}
\left(a_{1}, b_{1}, c_{1}\right) & \equiv\left\{\begin{array}{l}
\left(m_{1}, m_{1}+\omega_{1}, m_{1}+\alpha \omega_{1}\right) \\
\text { or }\left(m_{1}, m_{1}+p, m_{1}+2 p\right)
\end{array}\right. \\
\left(a_{2}, b_{2}, c_{2}\right) & \equiv\left\{\begin{array}{l}
\left(m_{2}, m_{2}+\omega_{2}, m_{2}+\alpha \omega_{2}\right) \\
\text { or }\left(m_{2}, m_{2}+p, m_{2}+2 p\right)
\end{array}\right. \\
\ldots, & \\
\left(a_{n}, b_{n}, c_{n}\right) & \equiv\left\{\begin{array}{l}
\left(m_{n}, m_{n}+\omega_{n}, m_{n}+\alpha \omega_{n}\right) \\
\text { or }\left(m_{n}, m_{n}+p, m_{n}+2 p\right) .
\end{array}\right.
\end{aligned}
$$

On eliminating the $m_{d}$ we have $2 n$ congruences modulo $3 p$ :

$$
\left(b_{i}-a_{i}, c_{i}-a_{i}\right) \equiv\left(\omega_{i}, \alpha \omega_{i}\right) \text { or }(p, 2 p), \quad i=1,2, \ldots, n \text {. }
$$

Since there are six permutations of $\left(a_{i}, b_{i}, c_{i}\right)$ and two possible congruences for each, there are $12^{n}$ possible sets of congruences represented by (3). (Although this number can be reduced somewhat, we prefer, for simplicity, to present the results of our original computations, which do not exploit additional symmetries in (3).) Thus by Lemma 2, if $S$ contains a Pasch, mitre, 6-cycle or crown configuration, there exists a $\mathcal{G} \in \Gamma$ and a corresponding set of congruences (3) which has, for some orderings of the blocks of $\mathcal{G}$ and some choice of the alternatives on the right of (3), a solution modulo 3 in which all the $\omega_{d}$ present satisfy $\omega_{d} \equiv 1(\bmod 3)$ and a solution modulo $p$ in which all the $\omega_{d}$ present satisfy $\theta\left(\omega_{d}\right)=1$. To show that this cannot happen, we examine each of the $12^{n}$ possible sets of congruences (3) for each configuration $\mathcal{G} \in \Gamma$. Denote this collection of congruence sets by $\Phi_{0}$. Thus $\left|\Phi_{0}\right|=12^{3}+3 \cdot 12^{4}+9 \cdot 12^{5}=2303424$.

As an immediate first step, we eliminate from $\Phi_{0}$ all sets where there are two intersecting blocks in the orbit of $\{0, p, 2 p\}$, for such configurations cannot occur in $S$. This leaves a collection $\Phi_{1}$ of 584064 sets: 864 for $\mathcal{G}_{1}, 7776$ each for $\mathcal{G}_{2}, \mathcal{G}_{3}$ and $\mathcal{G}_{4}, 62208$ each for $\mathcal{G}_{5}, \mathcal{G}_{6}, \ldots, \mathcal{G}_{13}$. For example, take the crown configuration $\mathcal{G}_{8}$. Denote the blocks other than $\{0,1, \alpha\}$, by $A, B, C, D$ and $E$, where $\{A, B\}$ and $\{C, D\}$ are pairs of parallel blocks. Then we have the following possibilities for blocks in the orbit of $\{0, p, 2 p\}$ : none, $6^{5}$; block $E, 6^{5}$; one or both of $\{A, B\}, 2 \cdot 6^{5}+6^{5}$; one or both of $\{C, D\}, 2 \cdot 6^{5}+6^{5}$. So the total number of legitimate congruence sets that arise from $\mathcal{G}_{8}$ is $8 \cdot 6^{5}=62208$. 
Next, we eliminate from $\Phi_{1}$ all cases where (3) has no solution modulo 3 . We assume that $\alpha=0$ and that $\omega_{d}=1$ for all multipliers $\omega_{d}$ present. We also assume that $p=1$. For if a set of congruences (3) has a solution modulo 3 with $p=2$ and includes the pairs $\left\{b_{j}-a_{j} \equiv p, c_{j}-a_{j} \equiv 2 p\right\}$ for those $j \in\{1,2, \ldots, n\}$ where the block $\left\{a_{j}, b_{j}, c_{j}\right\}$ is in the orbit of $\{0, p, 2 p\}$, then the set of congruences obtained by interchanging $b_{j}$ and $c_{j}$ has the same solution with $p=1$, and, of course, both sets of congruences are identical modulo $p$. After performing the computations we are left with the collection $\Phi_{2}$ of 3320 congruence sets, partitioned as follows: $\mathcal{G}_{1}, 32 ; \mathcal{G}_{2}, \mathcal{G}_{3}, \mathcal{G}_{4}, 168$ each; $\mathcal{G}_{5}, \mathcal{G}_{6}, \mathcal{G}_{7}, 384$ each; $\mathcal{G}_{8}, \mathcal{G}_{13}, 344$ each; $\mathcal{G}_{9}, \mathcal{G}_{12}, 224$ each; $\mathcal{G}_{10}, \mathcal{G}_{11}, 248$ each. In all cases the solution modulo 3 is unique.

We deal with $\Phi_{2}$ by examining each congruence set modulo $p$. For a given congruence set, let $t$ be the number of blocks in the orbit of $\{0, p, 2 p\}$ and note that $0 \leq t \leq 2$. Recall that the configuration has $n+1$ blocks. So there are $2 n$ congruences, $n$ point variables, $x_{1}, x_{2}, \ldots, x_{n}$, and $n-t$ multiplier variables, $\omega_{d}$. We select $2 n-t$ congruences by excluding $t$ (possibly none) of the $2 t$ congruences that involve $p$.

Suppose $t=0$. With the congruences (3) written in matrix form $\mathbf{D x}=\mathbf{e}$, we find that in every case $|\mathbf{D}|$ is a polynomial in $\alpha, d(\alpha)$, say, which is not identically zero. Assuming that $p$ is sufficiently large and $\alpha$ is chosen such that $d(\alpha) \not \equiv 0(\bmod p)$, we can obtain the unique solution $\mathbf{x}=\mathbf{D}^{-1} \mathbf{e}$ (modulo $p$ ), where $\mathbf{x}=\left(x_{1}, x_{2}, \ldots, x_{n}, \omega_{1}, \omega_{2}, \ldots, \omega_{n}\right)$ and the elements of $\mathbf{D}^{-1} \mathbf{e}$ are rational functions of $\alpha$. Next we attempt to compute the quadratic characters of the multipliers $\omega_{j}$ and the ratios $\omega_{j} / \omega_{k}$ on the assumption that $\theta(\lambda(\alpha))=1$ for each $\lambda(x) \in \Lambda$. In all except four cases we find that at least one multiplier or ratio of multipliers is not a quadratic residue modulo $p$, and hence $\mathcal{G}$ cannot occur in $S$. Example 1 illustrates this point. The remaining four cases correspond to a Pasch configuration and three 6 -cycles, where in the solution of the congruences (3) the $x_{i}$ are such that every block is of the form $\{a, b, c\}$ with $(a, b, c) \equiv(0,1, \alpha)(\bmod p)$ and the $\omega_{i}$ are all $\equiv 1(\bmod p)$. Since each $\omega_{i}$ is also congruent to 1 modulo 3 , it follows that each $\omega_{i}$ is equal to 1 . This in turn implies that the configuration contains repeated blocks. See Example 2 below.

Now suppose $t>0$. We find that it is always possible to choose $2 n-t$ congruences from (3) such that when they are written in matrix form $\mathbf{D} \mathbf{x}=\mathbf{e},|\mathbf{D}|=d(\alpha)$ is not identically zero. So if $\alpha$ is chosen such that $d(\alpha) \not \equiv 0(\bmod p)$, then we get a unique solution for the $2 n-t$ variables, $\mathbf{x}=\mathbf{D}^{-1} \mathbf{e}$. The excluded $t=1$ or 2 congruences have the form $b_{i}-a_{i} \equiv 0(\bmod p)$ or $c_{i}-a_{i} \equiv 0(\bmod p)$ for some $i$. So suppose for these $i$ that the solution $\mathbf{x}=\mathbf{D}^{-1} \mathbf{e}$ gives $a_{i}=a_{i}(\alpha), b_{i}=b_{i}(\alpha)$ and $c_{i}=c_{i}(\alpha)$ for rational functions $a_{i}(\alpha), b_{i}(\alpha)$ and $c_{i}(\alpha)$. We either have: (i) for all $t$ excluded congruences, $b_{i}(\alpha)-a_{i}(\alpha)$ or $c_{i}(\alpha)-a_{i}(\alpha)$ is identically zero for all $\alpha$; or (ii) for one of the excluded congruences there exists $\alpha$ such that $b_{i}(\alpha)-a_{i}(\alpha) \not \equiv 0(\bmod p)$ or $c_{i}(\alpha)-a_{i}(\alpha) \not \equiv 0(\bmod p)$.

In case (i), the excluded congruences may be ignored and we proceed as for $t=0$, where it turns out always that, assuming $\theta(\lambda(\alpha))=1$ for all $\lambda(x) \in \Lambda$, either $\theta\left(\omega_{j}\right)=-1$ for some multiplier $\omega_{j}$ or $\theta\left(\omega_{j} / \omega_{k}\right)=-1$ for some ratio $\omega_{j} / \omega_{k}$ of multipliers. Hence $\mathcal{G}$ does not occur in $S$. Example 3 illustrates this case.

In case (ii), by clearing the denominator we obtain an additional constraint, which takes the form $q(\alpha) \equiv 0(\bmod p)$ for some polynomial $q(x)$. Then if $\alpha$ is chosen such that $q(\alpha) \not \equiv 0(\bmod p)$, the congruences $(3)$ will be inconsistent and hence $\mathcal{G}$ will not occur in $S$. See Example 4.

To complete the proof we set $Q(x)$ equal to the least common multiple of all the determinant polynomials $d(x)$ and constraint polynomials $q(x)$ encountered in the preceding analysis. Observe that if $p$ is sufficiently large, none of the functions $d(x)$ and $q(x)$ depend 
on $p$, and hence $Q(x)$ is independent of $p$.

Whilst it is not feasible within the space limitations of this paper to give details of all the cases that occur in the proof of Lemma 5, the main features of the method can be illustrated by a few examples.

\section{Example 1.}

Let $\mathcal{G}$ be the 6 -cycle configuration $\mathcal{G}_{5}$ with the blocks ordered as written:

$$
\left\{\{0,1, \alpha\},\left\{0, x_{1}, x_{2}\right\},\left\{x_{5}, \alpha, x_{3}\right\},\left\{x_{3}, 0, x_{4}\right\},\left\{x_{1}, x_{5}, 1\right\},\left\{x_{4}, x_{2}, x_{5}\right\}\right\} .
$$

Suppose all these blocks belong to the orbit of $\{0,1, \alpha\}$. The congruences to be solved modulo $3 p$ are

$$
\begin{aligned}
\left(0, x_{1}, x_{2}\right) & \equiv\left(m_{1}, m_{1}+\omega_{1}, m_{1}+\alpha \omega_{1}\right), \\
\left(x_{5}, \alpha, x_{3}\right) & \equiv\left(m_{2}, m_{2}+\omega_{2}, m_{2}+\alpha \omega_{2}\right), \\
\left(x_{3}, 0, x_{4}\right) & \equiv\left(m_{3}, m_{3}+\omega_{3}, m_{3}+\alpha \omega_{3}\right), \\
\left(x_{1}, x_{5}, 1\right) & \equiv\left(m_{4}, m_{4}+\omega_{4}, m_{4}+\alpha \omega_{4}\right), \\
\left(x_{4}, x_{2}, x_{5}\right) & \equiv\left(m_{5}, m_{5}+\omega_{5}, m_{5}+\alpha \omega_{5}\right),
\end{aligned}
$$

or, after eliminating $m_{1}, m_{2}, m_{3}, m_{4}, m_{5}$,

$$
\begin{aligned}
\left(x_{1}, x_{2}\right) & \equiv\left(\omega_{1}, \alpha \omega_{1}\right), \\
\left(\alpha-x_{5}, x_{3}-x_{5}\right) & \equiv\left(\omega_{2}, \alpha \omega_{2}\right), \\
\left(-x_{3}, x_{4}-x_{3}\right) & \equiv\left(\omega_{3}, \alpha \omega_{3}\right), \\
\left(x_{5}-x_{1}, 1-x_{1}\right) & \equiv\left(\omega_{4}, \alpha \omega_{4}\right), \\
\left(x_{2}-x_{4}, x_{5}-x_{4}\right) & \equiv\left(\omega_{5}, \alpha \omega_{5}\right) .
\end{aligned}
$$

Setting $\alpha=0$ and $\omega_{1}=\omega_{2}=\omega_{3}=\omega_{4}=\omega_{5}=1$, we solve this set of congruences modulo 3 to obtain the unique solution:

$$
x_{1}=1, \quad x_{2}=0, \quad x_{3}=x_{4}=x_{5}=2 .
$$

Therefore we consider the congruences (4) modulo $p$, and for this purpose we put them into matrix form:

$$
\left[\begin{array}{rrrrrrrrrr}
-1 & 0 & 0 & 0 & 0 & 1 & 0 & 0 & 0 & 0 \\
0 & -1 & 0 & 0 & 0 & \alpha & 0 & 0 & 0 & 0 \\
0 & 0 & 0 & 0 & 1 & 0 & 1 & 0 & 0 & 0 \\
0 & 0 & -1 & 0 & 1 & 0 & \alpha & 0 & 0 & 0 \\
0 & 0 & 1 & 0 & 0 & 0 & 0 & 1 & 0 & 0 \\
0 & 0 & 1 & -1 & 0 & 0 & 0 & \alpha & 0 & 0 \\
1 & 0 & 0 & 0 & -1 & 0 & 0 & 0 & 1 & 0 \\
1 & 0 & 0 & 0 & 0 & 0 & 0 & 0 & \alpha & 0 \\
0 & -1 & 0 & 1 & 0 & 0 & 0 & 0 & 0 & 1 \\
0 & 0 & 0 & 1 & -1 & 0 & 0 & 0 & 0 & \alpha
\end{array}\right]\left[\begin{array}{c}
x_{1} \\
x_{2} \\
x_{3} \\
x_{4} \\
x_{5} \\
\omega_{1} \\
\omega_{2} \\
\omega_{3} \\
\omega_{4} \\
\omega_{5}
\end{array}\right] \equiv\left[\begin{array}{c}
0 \\
0 \\
\alpha \\
0 \\
0 \\
0 \\
0 \\
1 \\
0 \\
0
\end{array}\right](\bmod p)
$$

There are ten congruences and ten variables, the determinant of the system is $-\alpha\left(\alpha^{3}-\right.$ $\left.5 \alpha^{2}+6 \alpha-3\right)$, and we assume that $x\left(x^{3}-5 x^{2}+6 x-3\right)$ is a factor of $Q(x)$. Hence there is a unique solution modulo $p$ :

$$
x_{1}=\frac{\alpha^{4}-2 \alpha^{3}+3 \alpha-3}{\alpha^{3}-5 \alpha^{2}+6 \alpha-3}, x_{2}=\frac{\alpha^{5}-2 \alpha^{4}+3 \alpha^{2}-3 \alpha}{\alpha^{3}-5 \alpha^{2}+6 \alpha-3}, x_{3}=\frac{-\alpha^{4}+2 \alpha^{2}-2 \alpha}{\alpha^{3}-5 \alpha^{2}+6 \alpha-3},
$$




$$
\begin{gathered}
x_{4}=\frac{\alpha^{5}-\alpha^{4}-2 \alpha^{3}+4 \alpha^{2}-2 \alpha}{\alpha^{3}-5 \alpha^{2}+6 \alpha-3}, x_{5}=\frac{\alpha^{4}-3 \alpha^{3}+3 \alpha^{2}-2 \alpha}{\alpha^{3}-5 \alpha^{2}+6 \alpha-3}, \\
\omega_{1}=\frac{\alpha^{4}-2 \alpha^{3}+3 \alpha-3}{\alpha^{3}-5 \alpha^{2}+6 \alpha-3}, \omega_{2}=\frac{-2 \alpha^{3}+3 \alpha^{2}-\alpha}{\alpha^{3}-5 \alpha^{2}+6 \alpha-3}, \omega_{3}=\frac{\alpha^{4}-2 \alpha^{2}+2 \alpha}{\alpha^{3}-5 \alpha^{2}+6 \alpha-3}, \\
\omega_{4}=\frac{-\alpha^{3}+3 \alpha^{2}-5 \alpha+3}{\alpha^{3}-5 \alpha^{2}+6 \alpha-3}, \omega_{5}=\frac{-\alpha^{4}+2 \alpha^{3}-\alpha^{2}-\alpha}{\alpha^{3}-5 \alpha^{2}+6 \alpha-3} .
\end{gathered}
$$

Since $x, x-1,1-2 x$ and $-x^{3}+5 x^{2}-6 x+3$ are in $\Lambda$, we can assume that

$$
\theta(\alpha)=\theta(\alpha-1)=\theta(1-2 \alpha)=\theta\left(-\alpha^{3}+5 \alpha^{2}-6 \alpha+3\right)=1 .
$$

Hence we can compute the quadratic character of $\omega_{2}$,

$$
\theta\left(\omega_{2}\right)=\theta\left(\frac{-2 \alpha^{3}+3 \alpha^{2}-\alpha}{\alpha^{3}-5 \alpha^{2}+6 \alpha-3}\right)=\theta\left(\frac{\alpha(\alpha-1)(1-2 \alpha)}{\alpha^{3}-5 \alpha^{2}+6 \alpha-3}\right)=-1,
$$

and deduce that the configuration does not occur in $S$.

Example 2.

Let $\mathcal{G}$ be the Pasch configuration $\mathcal{G}_{1}$ with the blocks ordered as written:

$$
\left\{\{0,1, \alpha\},\left\{0, x_{1}, x_{2}\right\},\left\{x_{3}, 1, x_{2}\right\},\left\{x_{3}, x_{1}, \alpha\right\}\right\} .
$$

Suppose all these blocks belong to the orbit of $\{0,1, \alpha\}$. The congruences to be solved modulo $3 p$ are

$$
\begin{aligned}
& \left(0, x_{1}, x_{2}\right) \equiv\left(m_{1}, m_{1}+\omega_{1}, m_{1}+\alpha \omega_{1}\right), \\
& \left(x_{3}, 1, x_{2}\right) \equiv\left(m_{2}, m_{2}+\omega_{2}, m_{2}+\alpha \omega_{2}\right), \\
& \left(x_{3}, x_{1}, \alpha\right) \equiv\left(m_{3}, m_{3}+\omega_{3}, m_{3}+\alpha \omega_{3}\right),
\end{aligned}
$$

or, after eliminating $m_{1}, m_{2}, m_{3}$,

$$
\begin{aligned}
\left(x_{1}, x_{2}\right) & \equiv\left(\omega_{1}, \alpha \omega_{1}\right), \\
\left(1-x_{3}, x_{2}-x_{3}\right) & \equiv\left(\omega_{2}, \alpha \omega_{2}\right), \\
\left(x_{1}-x_{3}, \alpha-x_{3}\right) & \equiv\left(\omega_{3}, \alpha \omega_{3}\right) .
\end{aligned}
$$

Setting $\alpha=0$ and $\omega_{1}=\omega_{2}=\omega_{3}=1$, we solve this set of congruences modulo 3 to obtain the unique solution $x_{1}=1, x_{2}=x_{3}=0$. Therefore we consider the congruences (5) modulo $p$, and for this purpose we put them into matrix form:

$$
\left[\begin{array}{rrrrrr}
-1 & 0 & 0 & 1 & 0 & 0 \\
0 & -1 & 0 & \alpha & 0 & 0 \\
0 & 0 & 1 & 0 & 1 & 0 \\
0 & -1 & 1 & 0 & \alpha & 0 \\
-1 & 0 & 1 & 0 & 0 & 1 \\
0 & 0 & 1 & 0 & 0 & \alpha
\end{array}\right]\left[\begin{array}{l}
x_{1} \\
x_{2} \\
x_{3} \\
\omega_{1} \\
\omega_{2} \\
\omega_{3}
\end{array}\right] \equiv\left[\begin{array}{c}
0 \\
0 \\
1 \\
0 \\
0 \\
\alpha
\end{array}\right](\bmod p)
$$

The determinant of the system is $2 \alpha(\alpha-1)$, and we assume that $x(x-1)$ is a factor of $Q(x)$. Hence there is a unique solution modulo $p: x_{1}=1, x_{2}=\alpha, x_{3}=0, \omega_{1}=\omega_{2}=\omega_{3}=1$. 
In fact, this is one of the four configurations where the system of congruences (3) has a legitimate solution and, as previously explained, it does not exist in $S$.

The other three configurations where the congruences have legitimate solutions modulo $3 p$ are the 6 -cycles $\mathcal{G}_{5}, \mathcal{G}_{6}$ and $\mathcal{G}_{7}$, with the blocks, all in the orbit of $\{0,1, \alpha\}$, ordered as written:

$$
\begin{aligned}
& \mathcal{G}_{5}:\left\{\{0,1, \alpha\},\left\{0, x_{3}, x_{4}\right\},\left\{x_{5}, 1, x_{1}\right\},\left\{x_{5}, x_{2}, x_{4}\right\},\left\{0, x_{2}, x_{1}\right\},\left\{x_{5}, x_{3}, \alpha\right\}\right\}, \\
& \mathcal{G}_{6}:\left\{\{0,1, \alpha\},\left\{0, x_{5}, x_{1}\right\},\left\{x_{2}, x_{5}, x_{4}\right\},\left\{x_{3}, 1, x_{4}\right\},\left\{x_{2}, 1, x_{1}\right\},\left\{x_{3}, x_{5}, \alpha\right\}\right\}, \\
& \mathcal{G}_{7}:\left\{\{0,1, \alpha\},\left\{0, x_{1}, x_{5}\right\},\left\{x_{2}, x_{4}, x_{5}\right\},\left\{x_{3}, x_{4}, \alpha\right\},\left\{x_{2}, x_{1}, \alpha\right\},\left\{x_{3}, 1, x_{5}\right\}\right\} .
\end{aligned}
$$

Example 3.

Let $\mathcal{G}$ be the mitre configuration $\mathcal{G}_{2}$ with the blocks ordered as written:

$$
\left\{\{0,1, \alpha\},\left\{0, x_{1}, x_{2}\right\},\left\{x_{1}, x_{3}, 1\right\},\left\{x_{2}, x_{4}, \alpha\right\},\left\{0, x_{4}, x_{3}\right\}\right\} .
$$

Suppose the second, third and fourth blocks belong to the orbit of $\{0,1, \alpha\}$ and the fifth belongs to the orbit of $\{0, p, 2 p\}$. The congruences to be solved modulo $3 p$ are

$$
\begin{aligned}
\left(0, x_{1}, x_{2}\right) & \equiv\left(m_{1}, m_{1}+\omega_{1}, m_{1}+\alpha \omega_{1}\right), \\
\left(x_{1}, x_{3}, 1\right) & \equiv\left(m_{2}, m_{2}+\omega_{2}, m_{2}+\alpha \omega_{2}\right), \\
\left(x_{2}, x_{4}, \alpha\right) & \equiv\left(m_{3}, m_{3}+\omega_{3}, m_{3}+\alpha \omega_{3}\right), \\
\left(0, x_{4}, x_{3}\right) & \equiv\left(m_{4}, m_{4}+p, m_{4}+2 p\right),
\end{aligned}
$$

or, after eliminating $m_{1}, m_{2}, m_{3}, m_{4}$,

$$
\begin{aligned}
\left(x_{1}, x_{2}\right) & \equiv\left(\omega_{1}, \alpha \omega_{1}\right), \\
\left(x_{3}-x_{1}, 1-x_{1}\right) & \equiv\left(\omega_{2}, \alpha \omega_{2}\right), \\
\left(x_{4}-x_{2}, \alpha-x_{2}\right) & \equiv\left(\omega_{3}, \alpha \omega_{3}\right), \\
\left(x_{4}, x_{3}\right) & \equiv(p, 2 p) .
\end{aligned}
$$

Setting $\alpha=0$ and $\omega_{1}=\omega_{2}=\omega_{3}=p=1$, we solve this set of congruences modulo 3 to obtain this unique solution:

$$
x_{1}=1, \quad x_{2}=0, \quad x_{3}=2, \quad x_{4}=1 .
$$

Therefore we consider the congruences (6) modulo $p$, and for this purpose we put them into matrix form:

$$
\left[\begin{array}{rrrrrrr}
-1 & 0 & 0 & 0 & 1 & 0 & 0 \\
0 & -1 & 0 & 0 & \alpha & 0 & 0 \\
1 & 0 & -1 & 0 & 0 & 1 & 0 \\
1 & 0 & 0 & 0 & 0 & \alpha & 0 \\
0 & 1 & 0 & -1 & 0 & 0 & 1 \\
0 & 1 & 0 & 0 & 0 & 0 & \alpha \\
0 & 0 & 0 & -1 & 0 & 0 & 0 \\
0 & 0 & -1 & 0 & 0 & 0 & 0
\end{array}\right]\left[\begin{array}{c}
x_{1} \\
x_{2} \\
x_{3} \\
x_{4} \\
\omega_{1} \\
\omega_{2} \\
\omega_{3}
\end{array}\right] \equiv\left[\begin{array}{l}
0 \\
0 \\
0 \\
1 \\
0 \\
\alpha \\
0 \\
0
\end{array}\right](\bmod p)
$$

There are eight congruences but only seven variables. So we exclude the last congruence and work with just the first seven. The determinant of the reduced system is $\alpha^{2}(\alpha-1)$, 
and we assume that $x(x-1)$ is a factor of $Q(x)$. Hence there is a unique solution modulo $p$ :

$$
x_{1}=\frac{1}{1-\alpha}, x_{2}=\frac{\alpha}{1-\alpha}, x_{3}=x_{4}=0, \omega_{1}=\frac{1}{1-\alpha}, \omega_{2}=\frac{1}{\alpha-1}, \omega_{3}=\frac{\alpha}{\alpha-1}
$$

and, furthermore, this solution is consistent with the excluded congruence, $x_{3} \equiv 0(\bmod p)$. However, we can compute the quadratic character of $\omega_{1}$ :

$$
\theta\left(\omega_{1}\right)=\theta(1-\alpha)=-1
$$

since $x-1 \in \Lambda$, and therefore deduce that this configuration does not occur in $S$.

Example 4.

Let $\mathcal{G}$ be the crown configuration $\mathcal{G}_{8}$ with the blocks ordered as written:

$$
\left\{\{0,1, \alpha\},\left\{1, x_{1}, x_{4}\right\},\left\{x_{2}, x_{3}, x_{4}\right\},\left\{x_{1}, \alpha, x_{5}\right\},\left\{x_{3}, 0, x_{5}\right\},\left\{0, x_{2}, x_{1}\right\}\right\},
$$

and suppose only the last block belongs to the orbit of $\{0, p, 2 p\}$. The congruences to be solved modulo $3 p$ are

$$
\begin{aligned}
\left(1, x_{1}, x_{4}\right) & \equiv\left(m_{1}, m_{1}+\omega_{1}, m_{1}+\alpha \omega_{1}\right), \\
\left(x_{2}, x_{3}, x_{4}\right) & \equiv\left(m_{2}, m_{2}+\omega_{2}, m_{2}+\alpha \omega_{2}\right), \\
\left(x_{1}, \alpha, x_{5}\right) & \equiv\left(m_{3}, m_{3}+\omega_{3}, m_{3}+\alpha \omega_{3}\right), \\
\left(x_{3}, 0, x_{5}\right) & \equiv\left(m_{4}, m_{4}+\omega_{4}, m_{4}+\alpha \omega_{4}\right), \\
\left(0, x_{2}, x_{1}\right) & \equiv\left(m_{5}, m_{5}+p, m_{5}+2 p\right),
\end{aligned}
$$

or, after eliminating $m_{1}, m_{2}, m_{3}, m_{4}, m_{5}$,

$$
\begin{aligned}
\left(x_{1}-1, x_{4}-1\right) & \equiv\left(\omega_{1}, \alpha \omega_{1}\right), \\
\left(x_{3}-x_{2}, x_{4}-x_{2}\right) & \equiv\left(\omega_{2}, \alpha \omega_{2}\right), \\
\left(\alpha-x_{1}, x_{5}-x_{1}\right) & \equiv\left(\omega_{3}, \alpha \omega_{3}\right), \\
\left(-x_{3}, x_{5}-x_{3}\right) & \equiv\left(\omega_{4}, \alpha \omega_{4}\right), \\
\left(x_{2}, x_{1}\right) & \equiv(p, 2 p) .
\end{aligned}
$$

Setting $\alpha=0$ and $\omega_{1}=\omega_{2}=\omega_{3}=\omega_{4}=p=1$, this set of congruences has a unique solution modulo 3 :

$$
x_{1}=x_{3}=x_{5}=2, \quad x_{2}=x_{4}=1 .
$$

For solving modulo $p$, we put (7) in matrix form:

$$
\left[\begin{array}{rrrrrrrrr}
-1 & 0 & 0 & 0 & 0 & 1 & 0 & 0 & 0 \\
0 & 0 & 0 & -1 & 0 & \alpha & 0 & 0 & 0 \\
0 & 1 & -1 & 0 & 0 & 0 & 1 & 0 & 0 \\
0 & 1 & 0 & -1 & 0 & 0 & \alpha & 0 & 0 \\
1 & 0 & 0 & 0 & 0 & 0 & 0 & 1 & 0 \\
1 & 0 & 0 & 0 & -1 & 0 & 0 & \alpha & 0 \\
0 & 0 & 1 & 0 & 0 & 0 & 0 & 0 & 1 \\
0 & 0 & 1 & 0 & -1 & 0 & 0 & 0 & \alpha \\
0 & -1 & 0 & 0 & 0 & 0 & 0 & 0 & 0 \\
-1 & 0 & 0 & 0 & 0 & 0 & 0 & 0 & 0
\end{array}\right]\left[\begin{array}{l}
x_{1} \\
x_{2} \\
x_{3} \\
x_{4} \\
x_{5} \\
\omega_{1} \\
\omega_{2} \\
\omega_{3} \\
\omega_{4}
\end{array}\right] \equiv\left[\begin{array}{r}
-1 \\
-1 \\
0 \\
0 \\
\alpha \\
0 \\
0 \\
0 \\
0 \\
0
\end{array}\right](\bmod p)
$$


There are ten congruences but only nine variables. We temporarily remove the last congruence and consider only the first nine. However, the determinant of this reduced system is identically zero. So we take instead the first eight and the tenth congruences, omitting the ninth. This system has determinant $d(\alpha)=(\alpha-1)^{2}$, which is not zero modulo $p$ provided $x-1$ is a factor of $Q(x)$. The unique solution modulo $p$ of this system is then

$$
\begin{gathered}
x_{1}=0, x_{2}=\frac{1-2 \alpha+\alpha^{2}-\alpha^{3}}{(\alpha-1)^{2}}, x_{3}=\frac{-\alpha^{2}}{\alpha-1}, x_{4}=1-\alpha, x_{5}=\alpha^{2}, \\
\omega_{1}=-1, \omega_{2}=\frac{2 \alpha-1}{(\alpha-1)^{2}}, \omega_{3}=\alpha, \omega_{4}=\frac{\alpha^{2}}{\alpha-1},
\end{gathered}
$$

which is inconsistent with the omitted congruence, $x_{2} \equiv 0(\bmod p)$, unless $q(\alpha) \equiv$ $0(\bmod p)$, where $q(x)=1-2 x+x^{2}-x^{3}$. Since we can assume that $x-1$ and $q(x)$ are factors of $Q(x)$, this configuration does not occur in $S$.

Proof of Theorem 5. The result follows from Lemma 4 and Lemma 5. Choose $N$ greater than the degree of $Q(x)$. Take $p$ so large that it does not divide any of the coefficients of $Q(x)$ and is sufficiently large for Lemma 4 to apply. Then by Lemma 4 we select an $\alpha$ which is not a root of $Q(x)$ modulo $p$ and is such that $\theta(\lambda(\alpha))=1$ for all $\lambda(x) \in \Lambda$. If necessary we add a multiple of $p$ to $\alpha$ to obtain a value that is congruent to 0 modulo 3 . Now apply Lemma 5.

Finally, we briefly address a question which naturally arises. Can Theorem 4 be used to create 7-sparse Steiner triple systems? We suspect not. In our research we have been unable to find any 6-sparse system which avoids the 7-block, 9-point configuration $\{012$, $034,135,246,257,168,078\}$, obtained by adding a diagonal to the 'window frame'.

\section{References}

1. A. E. Brouwer, Steiner triple systems without forbidden subconfigurations, Mathematisch Centrum Amsterdam, ZW 104/77, 1977.

2. C. J. Colbourn, E. Mendelsohn, A. Rosa and J. Širáň, Anti-mitre Steiner triple systems, Graphs Combin. 10, 215-224 (1994).

3. P. Erdös, Problems and results in combinatorial analysis, Colloquio Internazionale sulle Teorie Combinatorie (Rome 1973), Tomo II, pp. 3-17. Atti dei Convegni Lincei, No. 17, Accad. Naz. Lincei, Rome 1976.

4. A. D. Forbes, M. J. Grannell and T. S. Griggs, On 6-sparse Steiner triple systems, J. Combin. Theory Ser. A 114, 235-252 (2007).

5. Y. Fujiwara, Constructions for anti-mitre Steiner triple systems, J. Combin. Des. 13, 286-291 (2005).

6. Y. Fujiwara, Infinite classes of anti-mitre and 5-sparse Steiner triple systems, J. Combin. Des. 14, 237-250 (2006).

7. M. J. Grannell, T. S. Griggs and C. A. Whitehead, The resolution of the anti-Pasch conjecture, J. Combin. Des. 8, 300-309 (2000). 
8. T. S. Griggs, J. P. Murphy and J. S. Phelan, Anti-Pasch Steiner triple systems, J. Combin. Inform. System Sci. 15, 79-84 (1990).

9. A. C. H. Ling, A direct product construction for 5-Sparse Steiner triple systems, J. Combin. Des. 5, 443-447 (1997).

10. A. C. H. Ling, C. J. Colbourn, M. J. Grannell and T. S. Griggs, Construction techniques for anti-Pasch Steiner triple systems, J. London Math. Soc. (2) 61, 641-657 (2000).

11. W. M. Schmidt, Equations over Finite Fields, Lecture Notes in Mathematics 536, Berlin Heidelberg New York: Springer 1976.

12. A. Wolfe, The resolution of the anti-mitre Steiner triple system conjecture, J. Combin. Des. 14, 229-236 (2006).

13. A. Wolfe, Private communication (2006).

14. A. Wolfe, 5 -sparse Steiner triple systems of order $n$ exist for almost all admissible $n$, Electron. J. Combin. 12, \#R68, 42 pp. (electronic) (2005).

15. A. Wolfe, The existence of 5 -sparse Steiner triple systems of order $n \equiv 3(\bmod 6), n \notin\{9,15\}$, J. Combin. Theory Ser. A, to appear (2008).

Received:

Final version received: 